\title{
REVIEW ARTICLE \\ Hand hygiene and risk of influenza virus infections in the community: a systematic review and meta-analysis
}

\author{
V.W. Y. WONG ${ }^{1}$, B. J. COWLING ${ }^{2}$ AND A. E. AIELLO ${ }^{3}$ \\ ${ }^{1}$ School of Nursing, Li Ka Shing Faculty of Medicine, The University of Hong Kong, Hong Kong Special \\ Administrative Region, China \\ ${ }^{2}$ Division of Epidemiology and Biostatistics, Li Ka Shing Faculty of Medicine, The University of Hong Kong, \\ Hong Kong Special Administrative Region, China \\ ${ }^{3}$ Department of Epidemiology, University of North Carolina Gillings School of Global Public Health, Chapel \\ Hill, NC, USA
}

Received 17 October 2013; Final revision 6 December 2013; Accepted 24 December 2013; first published online 23 January 2014

\section{SUMMARY}

Community-based prevention strategies for seasonal and pandemic influenza are essential to minimize their potential threat to public health. Our aim was to evaluate the efficacy of hand hygiene interventions in reducing influenza transmission in the community and to investigate the possible modifying effects of latitude, temperature and humidity on hand hygiene efficacy. We identified 979 articles in the initial search and 10 randomized controlled trials met our inclusion criteria. The combination of hand hygiene with facemasks was found to have statistically significant efficacy against laboratory-confirmed influenza while hand hygiene alone did not. Our meta-regression model did not identify statistically significant effects of latitude, temperature or humidity on the efficacy of hand hygiene. Our findings highlight the potential importance of interventions that protect against multiple modes of influenza transmission, and the modest efficacy of hand hygiene suggests that additional measures besides hand hygiene may also be important to control influenza.

Key words: Hygiene - personal, infectious disease control, influenza.

\section{INTRODUCTION}

Community-based prevention strategies for seasonal and pandemic influenza are essential to minimize their potential threat to public health $[1,2]$. Vaccination is the cornerstone of prevention of seasonal and pandemic influenza virus infections [3]. Although existing evidence demonstrates that vaccination can be an effective approach to protect the

\footnotetext{
* Author for correspondence: Dr B. J. Cowling, School of Public Health, Li Ka Shing Faculty of Medicine, The University of Hong Kong, Units 624-7, Core F, Cyberport 3, Pokfulam, Hong Kong.

(Email: bcowling@hku.hk)
}

population against influenza [4-6], uptake in some populations remains low [7-9]. In the event of a novel influenza pandemic, vaccines that provide good protection against the new strain might not be available for 4-6 months, and other control measures would be required in the interim, including nonpharmaceutical interventions such as hand hygiene [10]. Hand hygiene interventions are appealing because they can be applied in both developed and lesser developed regions at low cost $[10,11]$.

Influenza virus spreads among humans either by inhalation of virus-loaded droplets into the respiratory tract, by direct contact, e.g. hand shaking, or by indirect contact with infected individuals via contaminated 
objects (fomites) [12-14]. The relative importance of alternative modes of transmission is controversial, while the potential for efficacy of hand hygiene implicitly requires that direct or indirect contact is an important mode of transmission [15]. Recent research has suggested that the importance of contact transmission may vary in different regions [16]. For instance, ambient temperature and relative humidity may modify the mode of influenza transmission. Because small droplet transmission is enhanced by low or very high humidity [17], it has been hypothesized that in temperate zones with a cool and dry winter, influenza transmission is predominantly by aerosol while in tropical zones with a warm and humid environment, the virus is more often transmitted by the contact route [16]. If this hypothesis is correct, the effectiveness of hand hygiene interventions would be expected to vary by latitude, ambient temperature and humidity. If virus transmission in temperate zones primarily occurs by aerosol, then hand hygiene interventions would be expected to be less effective.

Since the World Health Organization highlighted the need for controlled trials in formulating the use of non-pharmaceutical interventions in preventing influenza transmission in 2006 [10], various randomized controlled trials (RCTs) and systematic reviews $[8,18]$ on the effectiveness of hand hygiene interventions in reducing influenza and other respiratory virus infections have been published. By contrast, there are three existing meta-analyses assessing the effectiveness of hand hygiene interventions in preventing respiratory diseases, none of which focused on influenza viruses specifically [19-21]. This systematic review and meta-analysis aims to evaluate the impact of hand hygiene interventions in preventing influenza virus transmission in the community setting and to investigate the possible modifying effects of latitude, temperature and humidity on hand hygiene efficacy for influenza virus infection.

\section{METHODS}

This meta-analysis was conducted in accordance with the Preferred Reporting Items for Systematic Reviews and Meta Analyses recommendations (PRISMA) statement [22].

\section{Search strategy}

We searched the Medline (January 1946 to November 2013), PubMed (January 1960 to November 2013),
EMBASE (1974 to November 2013), and Cochrane Library databases and the Cochrane Central Register of Controlled Trials (CENTRAL) (The Cochrane Library, 2013, Issue 11) databases using the following search terms in all fields regardless of publication date and language:

\#1: 'hand hygiene' OR 'hand washing' OR 'handwashing' OR 'hand-wash' OR 'hand sanitizers' OR 'hand sanitizer' OR 'hand rub'

\#2: 'influenza' OR 'flu' OR 'respiratory infection' OR 'respiratory virus' OR 'respiratory tract infection' OR 'respiratory illness' OR 'fever' OR 'cough' OR 'sore throat' OR 'runny nose' OR 'nasal congestion' OR 'sneezing' OR 'malaise' OR 'muscle aches' OR 'headache'

\#3: \#1 AND \#2

To identify further studies of interest, manual search was performed with the reference lists of retrieved review articles.

\section{Eligibility criteria}

We included any RCT comparing the effect of hand hygiene interventions with no intervention in reducing influenza virus transmission in community settings, in which study subjects or cluster units in a population were assigned prospectively into intervention and control groups using random allocation [23]. A community setting was defined as an open setting without confinement and special care for the participants. Articles describing any hand hygiene-related interventions alone were included.

\section{Study selection}

The primary outcome was the relative reduction of influenza virus infections confirmed by reversetranscriptase-polymerase chain reaction (RT-PCR), virus culture or rapid antigen test in the hand hygiene intervention group compared to the control group. The secondary outcome measure was the relative reduction of influenza-like illnesses (ILI) confirmed by either professional clinical diagnosis or reported symptoms. We adopted a febrile acute respiratory illness (FARI) definition which defines cases as the presence of fever with cough or sore throat [24].

Two independent reviewers (V.W.Y.C., B.J.C.) screened all titles of studies identified by the search strategy individually, then subsequently reviewed the abstracts of the potential relevant studies. If the 
studies described hand hygiene interventions and influenza transmission, the reviewers read the fulllength text. Further discussion was held if a consensus was not reached.

\section{Evidence quality assessment}

We evaluated the methodological quality of each outcome with GRADEprofiler (GRADEpro) [25], as recommended by the Cochrane Collaboration. We ranked the quality of evidence of each outcome as high, moderate, low, and very low based on its risk of bias, consistency, directness, precision of the results and publication bias.

\section{Statistical analysis}

The effect estimates were summarized as risk ratios (RRs) and their corresponding 95\% confidence intervals (CIs). Due to substantial variation in RRs, the summary statistic was estimated with the more conservative Mantel-Haenszel (MH) random-effects model since it accounts for both the potential variability in effects and also the random variability across studies associated with different study designs and settings. We assessed publication bias graphically with Begg's funnel plot [26] and also implemented Egger's test [27] and the Begg \& Mazumdar rank correlation [26] to quantify the evidence of publication bias statistically. For Egger's test, we considered evidence of publication bias if the two-tailed $P$ value was $<0 \cdot 05$. For rank correlation, we considered evidence of publication bias if the two-tailed $P$ value was $<0 \cdot 10$ since this test statistic has been shown to be less sensitive than Egger's test [28]. We calculated the $I^{2}$ statistic to assess the extent of inconsistency for each pooled estimate. The $I^{2}$ statistic quantified the proportion of total variations across effect estimates due to heterogeneity but not sampling error, and ranges from $0 \%$ to $100 \%$ such that $0 \%$ indicates homogeneity and $100 \%$ reflects substantial heterogeneity [29].

We performed separate analyses of studies in developed and developing countries due to their systematic differences such as cultural background, educational level, etc., and performed a subgroup analysis of hand hygiene interventions with or without facemask use for both outcomes. Meta-regression was conducted to further assess if any covariates could explain the variation across studies in the effect of hand hygiene on laboratory-confirmed influenza, i.e. the primary outcome. To test for a modifying impact of temperature and humidity on efficacy of hand hygiene, we constructed univariate random-effects regression models with a number of covariates including latitude, average temperature and humidity during studies. We calculated the mean of the average temperature and relative humidity during the recorded study months by using the data provided by WeatherSpark [30], which is a weather website summarizing historical data for the world from the National Oceanic and Atmospheric Administration. We carried out the meta-analysis using RevMan version 5.1 software [31] and the Comprehensive Meta-analysis version 2 software [32].

\section{RESULTS}

\section{Search results}

We identified 979 articles in the initial database search, of which 41 were retrieved based on their title and abstract content. Of the 41 retrieved articles, ten were eligible for meta-analysis based on our inclusion criteria (see Fig. 1). We excluded 31 studies after the full-length assessment [33-63] for the following reasons: studies were not RCTs, ineligible definition on ILI, no definition on respiratory diseases outcomes, hand hygiene interventions as a part of infection control programme, or no control group (see online Appendix). The characteristics of the ten eligible RCTs are summarized in Table 1, which comprised nine studies assessing laboratory-confirmed influenza [64-72] and ten studies assessing ILI [64-73].

\section{Quality of evidence}

The methodological qualities of studies were assessed by GRADEpro. Studies that used a laboratoryconfirmed influenza outcome were graded as high, while studies with an ILI-only outcome were graded as moderate. The evidence profile for each outcome is summarized in Table 2 (see also online Appendix). All included trials were RCTs with proper randomization and their allocation sequences were properly concealed. They were either single-blinded to the recruiting physician, principal investigator and statisticians or not blinded to any personnel. No significant publication bias was noted (see online Appendix). The imprecision was, however, significant in most of the trials due to small sample size, inadequate case ascertainment, poor compliance to interventions, 


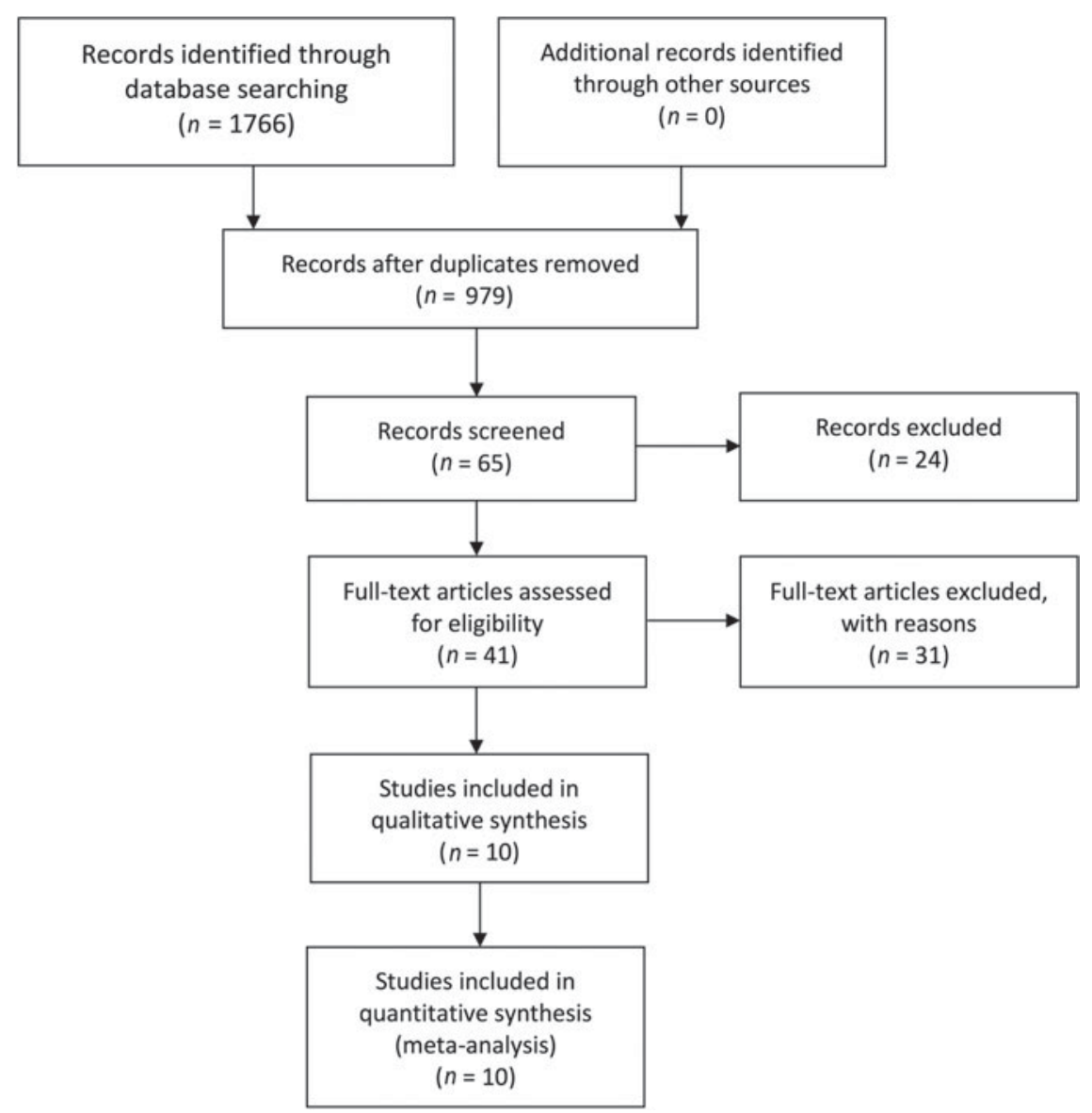

Fig. 1. Flow diagram of the process and results of study selection.

and insufficient statistical power. Most (8/10) of the studies received funding from the United States Centers for Disease Control and Prevention, one study was supported by the German Federal Ministry of Health, and one from a pharmaceutical company.

\section{Efficacy of hand hygiene interventions}

The forest plot for studies conducted in developed countries is shown in Figure 2. There was an insignificant relative risk reduction of $18 \%$ in the pooled analysis (RR $0 \cdot 82,95 \%$ CI $0 \cdot 66-1 \cdot 02, \quad I^{2}=0 \%$, $P=0.07$ ) of laboratory-confirmed influenza outcome. While a significant reduction of $27 \%$ was reported for the hand hygiene and facemask group (RR 0.73, $95 \%$ CI $\left.0 \cdot 53-0 \cdot 99, I^{2}=0 \%, P=0 \cdot 05\right)$, the hand hygiene only comparison was not statistically significant. A significant RR reduction of $22 \%$ (RR 0.78 , 95\% CI $0 \cdot 68-0 \cdot 90, I^{2}=0 \%, P=0 \cdot 0008$ ) was found in the pooled analysis of ILI outcomes. In the subgroup analyses, similar to the result from the laboratoryconfirmed influenza outcome, a significant reduction of $27 \% \quad\left(\mathrm{RR} \quad 0 \cdot 73,95 \%\right.$ CI $0.60-0 \cdot 89, I^{2}=0 \%$, $P=0.002)$ was noted for the combined comparison of hand hygiene and facemask use while the result from hand hygiene alone was not statistically significant.

There were only two studies in less developed countries. The efficacy of hand hygiene was not significant in the pooled analysis for the laboratory-confirmed influenza outcome. For the ILI outcome, a nonsignificant relative increase was observed for the efficacy of combined comparison of hand hygiene and facemask use (see online Appendix).

\section{Meta-regression}

We used meta-regression to explore if any particular covariate could explain the observed heterogeneity across studies (Table 3). A systematic review suggests that facemasks can reduce aerosol transmission of influenza virus [74]; therefore, we conducted metaregression on hand hygiene interventions without facemask to assess the independent effects of hand 
Table 1. Characteristics of included studies

\begin{tabular}{ll}
\hline \hline & $\begin{array}{l}\text { No. of studies } \\
(\%)\end{array}$ \\
Characteristics & \\
\hline Country & $8(80)$ \\
$\quad$ Developed & $2(20)$ \\
Developing & \\
Latitude (degrees) & $7(70)$ \\
$\quad \leqslant 23 \cdot 5$ & $3(30)$ \\
$>23 \cdot 5$ & \\
Setting & $5(50)$ \\
Household & $2(20)$ \\
Elementary school & $2(20)$ \\
University residential hall & $1(10)$ \\
Office & $6(60)$ \\
Transmission mode & $4(40)$ \\
Primary & \\
Secondary & $4(31)$ \\
Interventions evaluated* & $3(23)$ \\
Hand sanitizer and facemask & \\
Hand sanitizer, non-antibacterial soap & \\
$\quad$ and education & $2(15)$ \\
Hand sanitizer & $2(15)$ \\
Non-antibacterial soap and education & $1(8)$ \\
Non-antibacterial soap, education and & \\
$\quad$ facemask & \\
Hand sanitizer, non-antibacterial soap, & $1(8)$ \\
$\quad$ education and facemask & \\
Lutcome assessed* & $9(50)$ \\
Influenza-like-illness & $9(50)$ \\
\hline \hline
\end{tabular}

* Some studies assess more than one intervention and outcome.

hygiene even after adjusting for potential factors that could impact heterogeneity. For the studies conducted in developed countries, we found that a $10^{\circ}$ rise in latitude [relative risk ratio (RRR) $1 \cdot 28,95 \%$ CI $0.91-$ $1 \cdot 79, P=0 \cdot 15$ ], average temperature (RRR $0 \cdot 82,95 \%$ CI $0 \cdot 59-1 \cdot 13, P=0 \cdot 22)$ and average relative humidity (RRR 0.63, 95\% CI $0 \cdot 32-1 \cdot 22, P=0 \cdot 17$ ) were not statistically significantly associated with a change in the efficacy of hand hygiene in developed countries but the direction of the estimate for relative humidity was consistent with the hypothesis that influenza transmission is predominately by aerosol in temperate zones while the virus is commonly transmitted by contact route in tropical areas (see online Appendix).

\section{DISCUSSION}

We examined the efficacy of hand hygiene interventions in preventing influenza virus transmission in the community. The subgroup analysis from developed countries suggested that a combined intervention consisting of hand hygiene with facemasks is an effective strategy to prevent influenza, but we did not confirm the efficacy of hand hygiene alone for reducing influenza illness. This is consistent with evidence on the important role of aerosol transmission of influenza, such that interventions against contact transmission alone like hand hygiene may not be sufficient to control influenza transmission in the community [75]. However, shortcomings related to statistical power to detect the impact of hand hygiene suggest that future studies should continue to study the impact of hand hygiene independently on laboratoryconfirmed influenza outcomes.

Seasonal and pandemic influenza viruses cause a major burden of illness, hospitalization and death. Our review captured studies with the outcomes of laboratory-confirmed influenza or FARI (ILI) which is a fairly specific outcome to influenza. We did not include studies with broader definitions of respiratory illness, which could encompass many other outcomes such as other non-influenza viral infections, asthma exacerbation, allergic rhinitis or non-viral respiratory infections, because the efficacy of hand hygiene intervention on each respiratory illness might vary. According to these inclusion criteria, our review did not include studies that examined the efficacy of hand hygiene against broader respiratory illness outcomes, but these studies did identify reasonable efficacy of hand hygiene interventions [46, 54-56]. For this reason, this meta-analysis goes beyond three formerly published reviews [19-21] by focusing on influenza virus infections rather than any respiratory illness symptoms, and by exploring the hypothesis that modes of transmission may vary from region to region. In our meta-regression model, although we did not find any significant effects, we noted evidence for effects of all three covariates particularly from relative humidity. The insignificant result may due to relatively low sample size.

There are several noteworthy limitations in this review. The greatest limitation is the small number of RCTs that have been conducted to date on the efficacy of hand hygiene to control influenza. Since there are only a few studies involving the same hand hygiene interventions among the included studies, we are unable to provide intervention-specific pooled estimates. The efficacy of individual hand hygiene interventions, hence, cannot be compared. The heterogeneity across studies is another limitation and to address this 


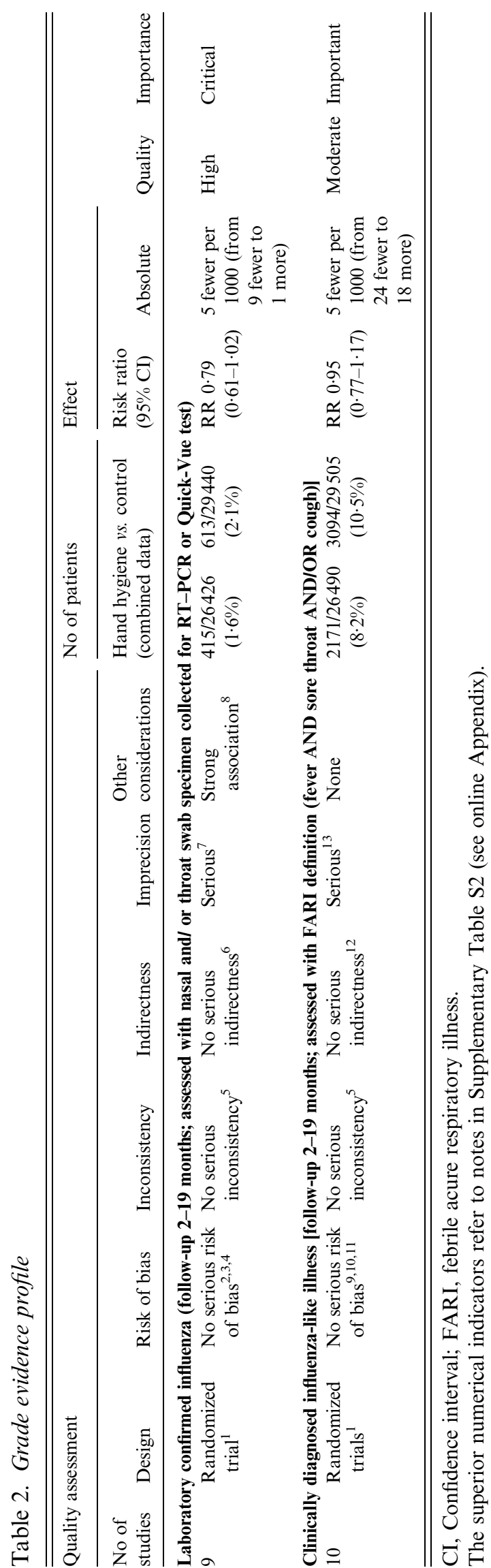

we performed separate analyses for developed and developing countries' data and meta-regression for hand hygiene only laboratory-confirmed influenza outcome. Although we cannot exclude the possible role of other covariates, we minimized the variations of different study design characteristics by including only RCTs. The variations associated with different settings and different hand hygiene interventions, however, cannot be ignored. The possible clustering effect may also be a limitation in our review. Since we did not adjust for clustering in the analysis, this may lead to skewed results with possibly higher risk of type I error and narrower confidence intervals. However, one previous study suggested that clustering effect did not have a significant effect on heterogeneity or overall pooled estimates from their meta-analysis assessing the effectiveness of hand hygiene interventions on infectious disease risk in the community setting [20].

The findings of this review have implications for the recommendations and guidelines of hand hygiene and facemask use in the future. Given the lack of substantial efficacy of hand hygiene identified in our review (Fig. 3), and the increasing evidence supporting a role of aerosol as a mode of influenza virus transmission [75-78], further public health initiatives may need to re-examine the control measures for aerosol transmission. In particular, measures such as hand hygiene that focus on reducing one mode of transmission (i.e. contact) may not be sufficient to control transmission. Measures that may require more detailed consideration include N95-type respirators, improved indoor ventilation, quarantining of infected individuals, and even the use of air humidifiers, given the potential role of humidity in reducing viability of aerosols $[16,17]$. While elucidating the possible influence of humidity in influenza transmission among human populations further confirms its contribution on influenza seasonality, particularly in temperate regions, the detailed mechanisms have yet to be explored.

The insignificant findings from hand hygiene intervention alone and subgroup analyses from developing countries' data does not necessarily indicate that hand hygiene is an ineffective measure for preventing influenza virus transmission. Rather, the non-significant results for hand hygiene alone could raise questions on compliance with existing recommendations on hand hygiene in the community. Indeed, hand washing and sanitizing needs to be practised properly and after all potential critical contamination events 


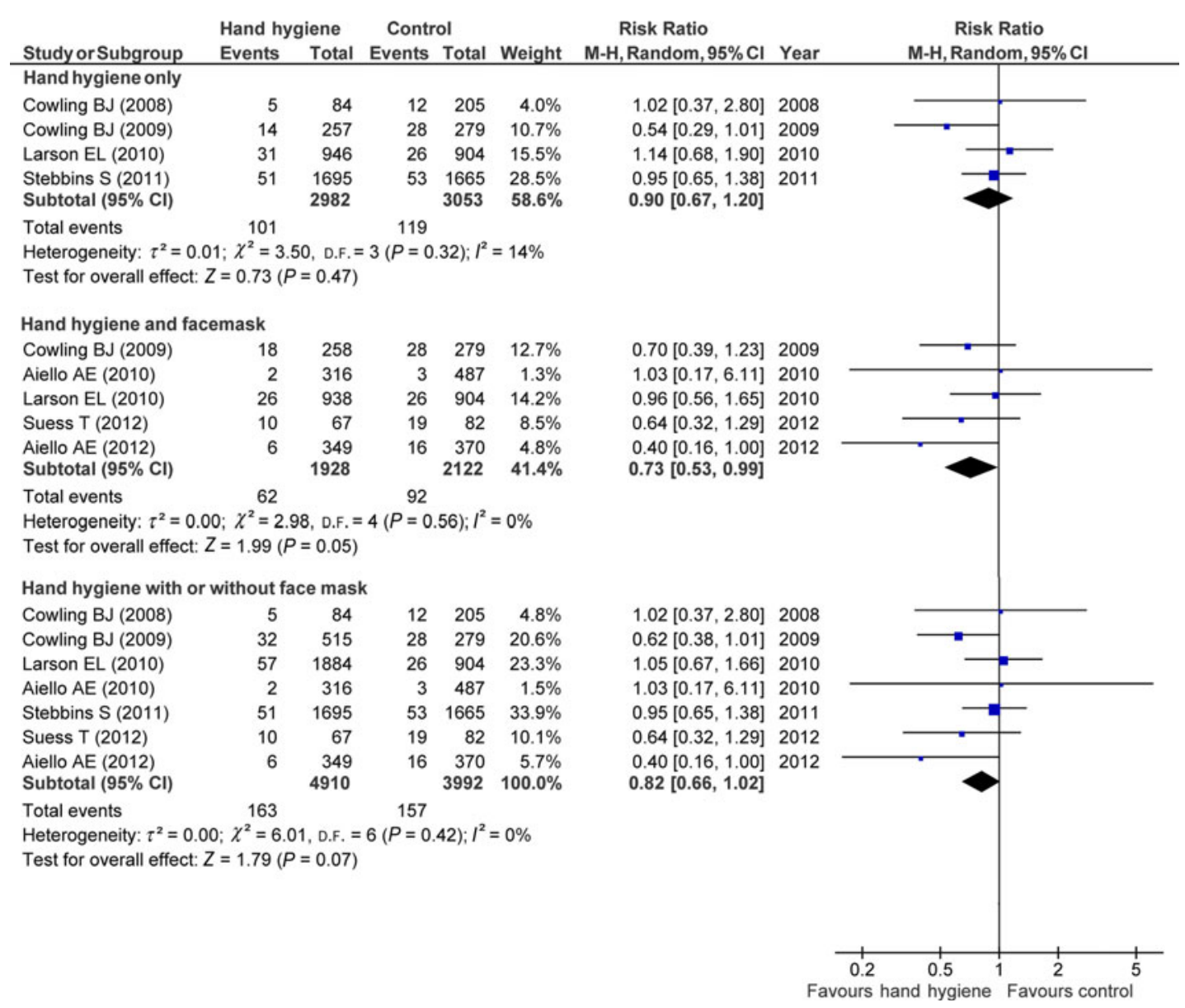

Fig. 2. Risk ratios for the effect of hand hygiene interventions with or without facemask on laboratory-confirmed influenza in studies conducted in developed countries.

that might occur throughout the day. The CDC recommends that individuals wash their hands with soap and water for at least 20 seconds, properly lathering hands, washing soap off, and drying hands completely or if a sink is not available, to use hand sanitizer when hands are not visibly soiled [15]. These recommendation are rarely carried out with high compliance in the general population [79]. Clearly, hand hygiene interventions not only need to be proven effective, but they also need to be widely adopted by most of the population if they are to mitigate influenza transmission effectively. Given the existing public health recommendations and guidelines on using hand hygiene interventions in preventing influenza transmission [11, 80, 81], the compliance rate in the community has not yet been well established. To our knowledge, there are only a few studies exploring interventions to promote hand hygiene practice in the community [82-86]. Further studies, in this regard, are warranted in relation to compliance rates of hand hygiene interventions and the possible interventions to promote such practices in the community.

In conclusion, hand hygiene interventions have been, and will continue to be an important component of the public health response to seasonal and pandemic influenza. However, expectations on the impact of such measures may need to be limited, given the results of our review indicating only potentially modest effects of this specific intervention. Variation in the importance of aerosol transmission in different regions is an intriguing possibility, and could imply the need for greater focus on alternative control measures particularly in temperate zones.

\section{SUPPLEMENTARY MATERIAL}

For supplementary material accompanying this paper visit http://dx.doi.org/10.1017/S095026881400003X. 
Table 3. Univariate regression analyses on different covariates in relation to the risk of laboratory-confirmed influenza in combined countries' data and developed countries' data (hand hygiene intervention only)

Laboratory-confirmed influenza (combined data - hand hygiene intervention only)

\begin{tabular}{|c|c|c|c|c|c|c|}
\hline \multirow[b]{2}{*}{ Covariates } & \multicolumn{3}{|c|}{ Combined data (six studies) } & \multicolumn{3}{|c|}{$\begin{array}{l}\text { Developed countries only } \\
\text { (four studies) }\end{array}$} \\
\hline & RRR & $95 \% \mathrm{CI}$ & $P$ value & RRR & $95 \% \mathrm{CI}$ & $P$ value \\
\hline Latitude $\left(10^{\circ}\right.$ change $)$ & $1 \cdot 00$ & $0 \cdot 66-1 \cdot 54$ & $0 \cdot 984$ & $1 \cdot 28$ & $0 \cdot 91-1 \cdot 79$ & $0 \cdot 145$ \\
\hline Average temperature $\left(10^{\circ} \mathrm{C}\right.$ change $)$ & $0 \cdot 93$ & $0 \cdot 59-1 \cdot 49$ & $0 \cdot 778$ & $0 \cdot 82$ & $0 \cdot 59-1 \cdot 13$ & $0 \cdot 221$ \\
\hline Average relative humidity ( 10 percentage point change) & $1 \cdot 20$ & $0 \cdot 89-1 \cdot 63$ & $0 \cdot 227$ & $0 \cdot 63$ & $0 \cdot 32-1 \cdot 22$ & $0 \cdot 169$ \\
\hline
\end{tabular}

RRR, Relative risk ratio; CI, confidence interval.

\begin{tabular}{|c|c|c|c|c|c|c|c|c|c|}
\hline \multirow{2}{*}{$\begin{array}{l}\text { Study or Subgroup } \\
\text { Hand hygiene only }\end{array}$} & $\begin{array}{l}\text { Hand hy } \\
\text { Events }\end{array}$ & $\begin{array}{l}\text { giene } \\
\text { Total }\end{array}$ & $\begin{array}{l}\text { Contr } \\
\text { Events }\end{array}$ & $\begin{array}{l}\text { ol } \\
\text { Total }\end{array}$ & \multicolumn{3}{|c|}{ Risk Ratio } & \multicolumn{2}{|c|}{$\begin{array}{c}\text { Risk Ratio } \\
\mathrm{M}-\mathrm{H}, \text { Random, } 95 \% \mathrm{Cl}\end{array}$} \\
\hline & & & & & & & & & \\
\hline Cowling BJ (2008) & 3 & 84 & 8 & 205 & $1.0 \%$ & $0.92[0.25,3.37]$ & 2008 & & \\
\hline Cowling BJ (2009) & 9 & 257 & 14 & 279 & $2.5 \%$ & $0.70[0.31,1.58]$ & 2009 & & \\
\hline Hübner NO (2010) & 3 & 64 & 3 & 65 & $0.7 \%$ & $1.02[0.21,4.85]$ & 2010 & & \\
\hline Larson EL (2010) & 101 & 946 & 113 & 904 & $26.5 \%$ & $0.85[0.66,1.10]$ & 2010 & & + \\
\hline $\begin{array}{l}\text { Stebbins S (2011) } \\
\text { Subtotal }(95 \% \mathrm{Cl})\end{array}$ & 72 & $\begin{array}{r}1695 \\
3046\end{array}$ & 80 & $\begin{array}{r}1665 \\
3118\end{array}$ & $\begin{array}{l}17.5 \% \\
48.2 \%\end{array}$ & $\begin{array}{l}0.88[0.65,1.21] \\
0.86[0.71,1.04]\end{array}$ & 2011 & & \\
\hline $\begin{array}{l}\text { Total events } \\
\text { Heterogeneity: } \tau^{2}=0 \\
\text { Test for overall effect: }\end{array}$ & $\begin{array}{c}188 \\
Z=1.59(P\end{array}$ & $\begin{array}{l}3, \text { D.F. }= \\
=0.11)\end{array}$ & \multicolumn{5}{|c|}{ Heterogeneity: $\tau^{2}=0.00 ; \chi^{2}=0.33$, D.F. $=4(P=0.99) ; I^{2}=0 \%$} & & \\
\hline \multicolumn{10}{|c|}{ Hand hygiene and facemask } \\
\hline Cowling BJ (2009) & 18 & 258 & 14 & 279 & $3.7 \%$ & $1.39[0.71,2.74]$ & 2009 & & \\
\hline Larson EL (2010) & 81 & 938 & 113 & 904 & $23.1 \%$ & $0.69[0.53,0.91]$ & 2010 & & \\
\hline Aiello AE (2010) & 40 & 367 & 80 & 552 & $13.4 \%$ & $0.75[0.53,1.07]$ & 2010 & & \\
\hline Aiello AE (2012) & 31 & 349 & 51 & 370 & $9.5 \%$ & $0.64[0.42,0.98]$ & 2012 & & \\
\hline $\begin{array}{l}\text { Suess T (2012) } \\
\text { Subtotal }(95 \% \mathrm{Cl})\end{array}$ & 6 & $\begin{array}{r}67 \\
1979\end{array}$ & 14 & $\begin{array}{r}82 \\
2187\end{array}$ & $\begin{array}{r}2.1 \% \\
51.8 \%\end{array}$ & $\begin{array}{l}0.52[0.21,1.29] \\
0.73[0.60,0.89]\end{array}$ & 2012 & & \\
\hline $\begin{array}{l}\text { Total events } \\
\text { Heterogeneity: } \tau^{2}=0 \\
\text { Test for overall effect: }\end{array}$ & $\begin{array}{c}176 \\
1 ; \chi^{2}=4.5 \\
Z=3.12(P\end{array}$ & $\begin{array}{l}1, \text { D.F. }= \\
=0.002\end{array}$ & $\begin{array}{r}272 \\
4(P=0 .\end{array}$ & $34) ; I^{2}=$ & $=11 \%$ & & & & \\
\hline \multicolumn{10}{|c|}{ Hand hygiene with or without facemask } \\
\hline Cowling BJ (2008) & 3 & 84 & 8 & 205 & $1.2 \%$ & $0.92[0.25,3.37]$ & 2008 & & \\
\hline Cowling BJ (2009) & 27 & 515 & 14 & 279 & $5.2 \%$ & $1.04[0.56,1.96]$ & 2009 & & \\
\hline Larson EL (2010) & 182 & 1884 & 113 & 904 & $41.7 \%$ & $0.77[0.62,0.96]$ & 2010 & & \\
\hline Hübner NO (2010) & 3 & 64 & 3 & 65 & $0.8 \%$ & $1.02[0.21,4.85]$ & 2010 & & \\
\hline Aiello AE (2010) & 40 & 367 & 80 & 552 & $16.1 \%$ & $0.75[0.53,1.07]$ & 2010 & & \\
\hline Stebbins S (2011) & 72 & 1695 & 80 & 1665 & $21.0 \%$ & $0.88[0.65,1.21]$ & 2011 & & \\
\hline Suess T (2012) & 6 & 67 & 14 & 82 & $2.5 \%$ & $0.52[0.21,1.29]$ & 2012 & & \\
\hline $\begin{array}{l}\text { Aiello AE }(2012) \\
\text { Subtotal }(95 \% \mathrm{Cl})\end{array}$ & 31 & $\begin{array}{r}349 \\
5025\end{array}$ & 51 & $\begin{array}{r}370 \\
4122\end{array}$ & $\begin{array}{r}11.5 \% \\
100.0 \%\end{array}$ & $\begin{array}{l}0.64[0.42,0.98] \\
0.78[0.68,0.90]\end{array}$ & 2012 & & \\
\hline \multicolumn{10}{|c|}{$\begin{array}{l}\text { Total events } \quad 364 \\
\text { Heterogeneity: } \tau^{2}=0.00 ; \chi^{2}=3.20, \text { D.F. }=7(P= \\
\text { Test for overall effect: } Z=3.36(P=0.0008)\end{array}$} \\
\hline
\end{tabular}

Fig. 3. Risk ratios for the effect of hand hygiene interventions with or without facemask on influenza-like illness in studies conducted in developed countries.

\section{ACKNOWLEDGEMENTS}

This work was supported by the Harvard Center for Communicable Disease Dynamics from the National
Institute of General Medical Sciences (grant no. U54 GM088558), and the Area of Excellence Scheme of the Hong Kong University Grants Committee (grant no. AoE/M-12/06). 


\section{DECLARATION OF INTEREST}

B.J.C. received research funding from MedImmune Inc., and consults for Crucell NV. A.E.A. consults for SCA Tork as part of the Tork Green Hygiene Council.

\section{REFERENCES}

1. Molinari N-AM, et al. The annual impact of seasonal influenza in the US: measuring disease burden and costs. Vaccine 2007; 25: 5086-5096.

2. Carcione D, et al. Secondary attack rate of pandemic influenza A(H1N1) 2009 Western Australian households, 29 May-7 August 2009. Eurosurveillance 2011; 16: pii $=19765$.

3. Fiore AE, et al. Prevention and control of influenza with vaccines: recommendations of the Advisory Committee on immunization practices (ACIP). Atlanta, US: The Centers for Disease Control and Prevention (CDC), 2010 .

4. Jefferson $\mathbf{T}$, et al. Vaccines for preventing influenza in healthy adults. Cochrane Database of Systematic Reviews 2010. Issue no. 7. Art. no. CD001269.

5. Jefferson $\mathbf{T}$, et al. Vaccines for preventing influenza in healthy children. Cochrane Database of Systematic Reviews 2008. Issue no. 2. Art. no. CD004879.

6. Ohmit SE, et al. Prevention of antigenically drifted influenza by inactivated and live attenuated vaccines. New England Journal of Medicine 2006; 355: 2513-2522.

7. Bohmer MM, et al. Barriers to pandemic influenza vaccination and uptake of seasonal influenza vaccine in the post-pandemic season in Germany. BMC Public Health 2012; 12: 938.

8. Warren-Gash C, Fragaszy E, Hayward AC. Hand hygiene to reduce community transmission of influenza and acute respiratory tract infection: a systematic review. Influenza and Other Respiratory Viruses 2013; 7: 738-749.

9. Sammon CJ, et al. Factors associated with uptake of seasonal and pandemic influenza vaccine among clinical risk groups in the UK: an analysis using the General Practice Research Database. Vaccine 2011; 20: 2483-2489.

10. Bell DM, World Health Organization Writing Group. Non-pharmaceutical interventions for pandemic influenza, national and community measures. Emerging Infectious Diseases 2006; 12: 88-94.

11. Smith NM, et al. Prevention and control of influenza: recommendations of the Advisory Committee on Immunization Practices (ACIP). Atlanta: The Centers for Disease Control and Prevention, 2006.

12. Brankston G, et al. Transmission of influenza A in human beings. Lancet Infectious Diseases 2007; 7: 257-265.

13. Tellier R. Aerosol transmission of influenza A virus: a review of new studies. Journal of The Royal Society 2009; 6 (Suppl. 6): S783-790.
14. Weber TP, Stilianakis NI. Inactivation of influenza A viruses in the environment and modes of transmission: a critical review. Journal of Infection 2008; 57: 361-373.

15. Boyce JM, et al. Guideline for hand hygiene in healthcare settings. Recommendations of the Healthcare Infection Control Practices Advisory Committee and the HICPAC/SHEA/APIC/IDSA Hand Hygiene Task Force. Society for Healthcare Epidemiology of America/Association for Professionals in Infection Control/Infectious Diseases Society of America. Morbidity and Mortality Weekly Report. Recommendations and Reports/Centers for Disease Control 2002; 51: 1-45, quiz CE41-44.

16. Lowen A, Palese P. Transmission of influenza virus in temperate zones is predominantly by aerosol, in the tropics by contact: a hypothesis. PLoS Currents 2009; 1: RRN1002.

17. Yang W, Marr LC. Dynamics of airborne influenza A viruses indoors and dependence on humidity. PLoS One 2011; 6: e21481.

18. Jefferson T, et al. Physical interventions to interrupt or reduce the spread of respiratory viruses. Cochrane Database of Systematic Reviews 2011. Issue no. 7. Art. no. CD006207.

19. Aiello AE, Larson EL. What is the evidence for a causal link between hygiene and infections? Lancet Infectious Diseases 2002; 2: 103-110.

20. Aiello AE, et al. Effect of hand hygiene on infectious disease risk in the community setting: a metaanalysis. Americal Journal of Public Health 2008; 98: 1372-1381.

21. Rabie T, Curtis V. Handwashing and risk of respiratory infections: a quantitative systematic review. Tropical Medicine and International Health 2006; 11: 258-267.

22. Moher D, et al. Preferred reporting items for systematic reviews and meta-analyses: The PRISMA Statement. PLoS Medicine 2009; 6: e1000097.

23. A Dictionary of Epidemiology. 5th edn. New York, US: Oxford University Press, 2008.

24. Babcock HM, Merz LR, Fraser VJ. Is influenza an influenza-like illness? Clinical presentation of influenza in hospitalized patients. Infection Control and Hospital Epidemiology 2006; 27: 266-270.

25. Brozek J, Oxman A, Schünemann H. Version 3.6 for Windows 2008.

26. Begg CB, Berlin JA. Publication bias and dissemination of clinical research. Journal of the National Cancer Institute 1989; 81: 107-115.

27. Egger M, et al. Bias in meta-analysis detected by a simple, graphical test. British Medical Journal 1997; 315: 629-634

28. Sterne JAC, Gavaghan D, Egger M. Publication and related bias in meta-analysis: power of statistical tests and prevalence in the literature. Journal of Clinical Epidemiology 2000; 53: 1119-1129.

29. Higgins JP, et al. Measuring inconsistency in metaanalyses. British Medical Journal 2003; 327: 557-560.

30. Diebel J, Norda J. Bay Area, California, the United State of America: WeatherSpark, 2012 (http://weather spark.com/). Accessed 26 November 2012. 
31. Review Manager (RevMan). Version 5.1 [computer program]. Copenhagen: The Nordic Cochrane Centre, The Cochrane Collaboration, 2011.

32. Borenstein M, et al. Comprehensive Meta-analysis version 2, Biostat [computer program]. Englewood, NJ, 2005.

33. Apisarnthanarak A, et al. Intervention with an infection control bundle to reduce transmission of influenza-like illnesses in a Thai preschool. Infection Control and Hospital Epidemiology 2009; 30: 817-822.

34. Castilla $\mathbf{J}$, et al. Risk factors and effectiveness of preventive measures against influenza in the community. Influenza and Other Respiratory Viruses 2013; 7: 177183.

35. Dyer DL, Shinder A, Shinder F. Alcohol-free instant hand sanitizer reduces elementary school illness absenteeism. Family Medicine 2000; 32: 633-638.

36. Falsey AR, et al. Evaluation of a handwashing intervention to reduce respiratory illness rates in senior daycare centers. Infection Control and Hospital Epidemiology 1999; 20: 200-202.

37. Gautret $\mathbf{P}$, et al. Protective measures against acute respiratory symptoms in French pilgrims participating in the Hajj of 2009. Journal of Travel Medicine 2011; 18: 53-55.

38. Godoy P, et al. Effectiveness of hand hygiene and provision of information in preventing influenza cases requiring hospitalization. Preventive Medicine 2012; 54: $434-439$.

39. Kotch JB, et al. Evaluation of an hygienic intervention in child day-care centers. Pediatrics 1994; 94: 991-994.

40. Krilov LR, et al. Impact of an infection control program in a specialized preschool. American Journal of Infection Control 1996; 24: 167-173.

41. Ladegaard MB, Stage V. Hand-hygiene and sickness among small children attending day care centers. An intervention study [in Danish]. Ugeskrift for Lager 1999; 161: 4396-4400.

42. Larson EL, et al. Effect of antibacterial home cleaning and handwashing products on infectious disease symptoms: a randomized, double-blind trial. Annals of Internal Medicine 2004; 140: 321-329.

43. Lau CH, et al. Hand hygiene instruction decreases illness-related absenteeism in elementary schools: a prospective cohort study. BMC Pediatrics 2012; 12: 52.

44. Liu WT, et al. A case-control study of the transmission of pandemic influenza A (H1N1) virus in families [in Chinese]. Zhonghua Jie He He Hu Xi Za Zhi 2011; 34: 509-514.

45. Loustalot F, et al. Household transmission of 2009 pandemic influenza a (H1N1) and nonpharmaceutical interventions among households of high school students in San Antonio, Texas. Clinical Infectious Diseases 2011; 52: S146-S153.

46. Luby SP, et al. Effect of handwashing on child health: a randomised controlled trial. Lancet 2005; 366: 225233.

47. Luby SP, et al. Using child health outcomes to identify effective measures of handwashing. American Journal of Tropical Medicine and Hygiene 2011; 85: 882-892.
48. Master D, Hess Longe SH, Dickson H. Scheduled hand washing in an elementary school population. Family Medicine 1997; 29: 336-339.

49. Mitchell T, et al. Non-pharmaceutical interventions during an outbreak of 2009 pandemic influenza A (H1N1) virus infection at a large public university, April-May 2009. Clinical Infectious Diseases 2011; 52 (Suppl. 1): S138-145.

50. Morton JL, Schultz AA. Healthy Hands: Use of alcohol gel as an adjunct to handwashing in elementary school children. Journal of School Nursing 2004; 20: 161-167.

51. Nandrup-Bus I. Mandatory handwashing in elementary schools reduces absenteeism due to infectious illness among pupils: a pilot intervention study. American Journal of Infection Control 2009; 37: 820-826.

52. Niffenegger JP. Proper handwashing promotes wellness in child care. Journal of Pediatric Health Care 1997; 11: 26-31.

53. Pandejpong D, et al. Appropriate time-interval application of alcohol hand gel on reducing influenza-like illness among preschool children: a randomized, controlled trial. American Journal of Infection Control 2012; 40: 507-511.

54. Roberts L, et al. Effect of infection control measures on the frequency of upper respiratory infection in child care: a randomized, controlled trial. Pediatrics 2000; 105: 738-742.

55. Sandora TJ, Shih MC, Goldmann DA. Reducing absenteeism from gastrointestinal and respiratory illness in elementary school students: a randomized, controlled trial of an infection-control intervention. Pediatrics 2008; 121: e1555-1562.

56. Sandora TJ, et al. A randomized, controlled trial of a multifaceted intervention including alcohol-based hand sanitizer and hand-hygiene education to reduce illness transmission in the home. Pediatrics 2005; 116: 587-594.

57. Savolainen-Kopra C, et al. Hand washing with soap and water together with behavioural recommendations prevents infections in common work environment: an open cluster-randomized trial. Trials 2012; 13: 10 .

58. Thumma J, Aiello AE, Foxman B. The association between handwashing practices and illness symptoms among college students living in a university dormitory. American Journal of Infection Control 2009; 37: 70-72.

59. Tsalik EL, et al. An infection control program for a 2009 influenza A H1N1 outbreak in a university-based summer camp. Journal of American College Health 2011; 59: 419-426.

60. White C, et al. The impact of a health campaign on hand hygiene and upper respiratory illness among college students living in residence halls. Journal of American College Health 2005; 53: 175-181.

61. White C, et al. The effect of hand hygiene on illness rate among students in university residence halls. American Journal of Infection Control 2003; 31: 364 370.

62. White CG, et al. Reduction of illness absenteeism in elementary schools using an alcohol-free instant hand sanitizer. Journal of School Nursing 2001; 17: 258-265. 
63. Miller JR, et al. Use of nonpharmaceutical interventions to reduce transmission of 2009 pandemic influenza A (pH1N1) in Pennsylvania public schools. Journal of School Health 2013; 83: 281-289.

64. Aiello AE, et al. Mask use, hand hygiene, and seasonal influenza-like illness among young adults: a randomized intervention trial. Journal of Infectious Diseases 2010; 201: 491-498.

65. Aiello AE, et al. Facemasks, hand hygiene, and influenza among young adults: A randomized intervention trial. PLoS One 2012; 7: e29744.

66. Cowling BJ, et al. Facemasks and hand hygiene to prevent influenza transmission in households: a cluster randomized trial. Annals of Internal Medicine 2009; 151: 437-446.

67. Cowling BJ, et al. Preliminary findings of a randomized trial of non-pharmaceutical interventions to prevent influenza transmission in households. PLoS One 2008; 3: e2101.

68. Larson EL, et al. Impact of non-pharmaceutical interventions on URIs and influenza in crowded, urban households. Public Health Reports 2010; 125: 178-191.

69. Simmerman JM, et al. Findings from a household randomized controlled trial of hand washing and face masks to reduce influenza transmission in Bangkok, Thailand. Influenza and Other Respiratory Viruses 2011; 5: 256-267.

70. Suess T, et $\boldsymbol{a l}$. Facemasks and intensified hand hygiene in a German household trial during the 2009/2010 influenza $\mathrm{A}(\mathrm{H} 1 \mathrm{~N} 1)$ pandemic: adherence and tolerability in children and adults. Epidemiology and Infection 2011; 139: 1895-1901.

71. Talaat M, et al. Effects of hand hygiene campaigns on incidence of laboratory-confirmed influenza and absenteeism in schoolchildren, Cairo, Egypt. Emerging Infectious Diseases 2011; 17: 619-625.

72. Stebbins $\mathbf{S}$, et al. Reduction in the incidence of influenza A but not influenza B associated with use of hand sanitizer and cough hygiene in schools: a randomized controlled trial. Pediatric Infectious Disease Journal 2011; 30: 921-926.

73. Hubner NO, et al. Effectiveness of alcohol-based hand disinfectants in a public administration: impact on health and work performance related to acute respiratory symptoms and diarrhoea. BMC Infectious Diseases 2010; 10: 250 .
74. Cowling BJ, et al. Face masks to prevent transmission of influenza virus: a systematic review. Epidemiology and Infection 2010; 138: 449-456.

75. Cowling BJ, et al. Aerosol transmission is an important mode of influenza A virus spread. Nature Communications 2013; 4: 1935.

76. Killingley B, Nguyen-Van-Tam J. Routes of influenza transmission. Influenza and Other Respiratory Viruses 2013; 7 (Suppl. 2): 42-51.

77. Milton DK, et al. Influenza virus aerosols in human exhaled breath: particle size, culturability, and effect of surgical masks. Public Library of Science Pathogens 2013; 9: e1003205.

78. Bischoff WE, et al. Exposure to influenza virus aerosols during routine patient care. Journal of Infectious Diseases 2013; 207: 1037-1046.

79. American Society for Microbiology. American Society for Microbiology survey reveals that as many as 30 percent of travelers don't wash hands after using public restrooms at airports Chicago, US: American Society for Microbiology, 2003.

80. Department of Health. Save lives: clean your hands, 2012 (http://www.dh.gov.uk/health/2012/05/save-livesclean-your-hands/). Accessed 12 June 2012.

81. Centre for Health Protection. CHP investigates influenzalike illness outbreak, 2012 (http://www.chp.gov.hk/en/ view_content/25291.html). Accessed 12 June 2012.

82. Yardley L, et al. Evaluation of a Web-based intervention to promote hand hygiene: exploratory randomized controlled trial. Journal of Medical Internet Research 2011; 13: e107.

83. Bourgeois FT, et al. Evaluation of influenza prevention in the workplace using a personally controlled health record: randomized controlled trial. Journal of Medical Internet Research 2008; 10: e5.

84. Curtis V, de Barra M, Aunger R. Disgust as an adaptive system for disease avoidance behaviour. Philosophical Transactions of the Royal Society of London. Series B, Biological Sciences 2011; 366: 389-401.

85. Hubner NO, Hubner C, Kramer A. Impact of health campaign on hand hygiene with alcohol-based hand rubs in a non-clinical setting. Journal of Hospital Infection 2013; 83 (Suppl. 1): S23-28.

86. Zomer TP, et al. Hand hygiene compliance and environmental determinants in child day care centers: an observational study. American Journal of Infection Control 2013; 41: 497-502. 